\title{
Directivity analysis of meander-line-coil EMATs with a wholly analytical method
}

DOI:

10.1016/j.ultras.2016.09.016

\section{Document Version}

Accepted author manuscript

Link to publication record in Manchester Research Explorer

\section{Citation for published version (APA):}

Xie, Y., Liu, Z., Yin , L., Deng, P., \& Yin, W. (2017). Directivity analysis of meander-line-coil EMATs with a wholly analytical method. Ultrasonics, 73, 262-270. https://doi.org/10.1016/j.ultras.2016.09.016

\section{Published in:}

Ultrasonics

\section{Citing this paper}

Please note that where the full-text provided on Manchester Research Explorer is the Author Accepted Manuscript or Proof version this may differ from the final Published version. If citing, it is advised that you check and use the publisher's definitive version.

\section{General rights}

Copyright and moral rights for the publications made accessible in the Research Explorer are retained by the authors and/or other copyright owners and it is a condition of accessing publications that users recognise and abide by the legal requirements associated with these rights.

\section{Takedown policy}

If you believe that this document breaches copyright please refer to the University of Manchester's Takedown Procedures [http://man.ac.uk/04Y6Bo] or contact uml.scholarlycommunications@manchester.ac.uk providing relevant details, so we can investigate your claim.

\section{OPEN ACCESS}




\title{
Directivity analysis of Meander-Line-Coil EMATs with a wholly analytical method
}

\author{
Yuedong Xie ${ }^{\mathrm{a}}$, Zenghua Liu ${ }^{\mathrm{b}}$, Liyuan Yin ${ }^{\mathrm{c}}$, Jiande ${ }^{\mathrm{c}}$, Wuc Peng Deng ${ }^{\mathrm{b}}$, Wuliang Yin ${ }^{\mathrm{a},{ }^{*}}$ \\ ${ }^{\text {a }}$ School of Electrical and Electronic Engineering, University of Manchester; Manchester, United Kingdom \\ ${ }^{\mathrm{b}}$ College of Mechanical Engineering and Applied Electronics Technology, Beijing University of Technology, \\ Beijing, 100124, China. \\ ${ }^{c}$ School of Information Engineering and Automation, Kunming University of Science and Technology, \\ Kunming, China \\ * Corresponding Author: E-mail: wuliang.yin@manchester.ac.uk; Fax number: +44 (0) 161306 4644; \\ Telephone number: +44 (0) 161 -3062885
}

\begin{abstract}
This paper presents the simulation and experimental study of the radiation pattern of a meander-line-coil EMAT. A wholly analytical method, which involves the coupling of two models: an analytical EM model and an analytical UT model, has been developed to build EMAT models and analyse the Rayleigh waves' beam directivity. For a specific sensor configuration, Lorentz forces are calculated using the EM analytical method, which is adapted from the classic Deeds and Dodd solution. The calculated Lorentz force density are imported to an analytical ultrasonic model as driven point sources, which produce the Rayleigh waves within a layered medium. The effect of the length of the meander-line-coil on the Rayleigh waves' beam directivity is analysed quantitatively and verified experimentally.
\end{abstract}

Keywords: Electromagnetic acoustic transducers (EMATs), Rayleigh waves, Modelling and simulation, Analytical solutions, Beam directivity

\section{Introduction}

A variety of non-destructive testing (NDT) techniques are available in industries, such as radiographic testing (RT), magnetic particle testing (MPT), eddy current methods, magnetic flux leakage (MFL) and ultrasonic methods [1-5]. Due to its advantages of good penetration depth and mechanical flexibility, the piezoelectric ultrasonic method is widely used for thickness measurement, flaw evaluation and material characterization [6-11]. The transducer frequently used for the conventional ultrasonic non-destructive testing is piezoelectric ceramics or crystals [10-12]. However, one primary disadvantage of the piezoelectric ultrasonic testing is the need to have good sonic contact with the test piece, typically by means of a couplant for acoustic impedance matching [13]. Because an EMAT generates ultrasonic waves directly into the testing piece instead of coupling through the transducer, it has advantages in applications where surface contact is not possible or desirable [14, 15]. Another attractive feature of EMAT is that a variety of waves modes can be produced based on different combinations of coils and magnets $[16,17]$.

There are two EMAT interactions which can produce ultrasound: the magnetostriction mechanism and the Lorentz force mechanism; both the magnetostriction mechanism and the Lorentz force mechanism are for ferromagnetic materials while only the Lorentz force mechanism is for conductive metallic materials $[16,18,19]$. In this paper, only EMAT based on Lorentz force mechanism to generate Rayleigh waves is discussed. As shown in Figure 1, an EMAT system consists basically of a coil carrying an alternating current, a permanent magnet providing a large static magnetic field, and the test piece. In this work, the test piece used is an aluminium plate, which is mainly affected by Lorentz force mechanism, so 
magnetostriction is not considered. The Lorentz force mechanism is: the coil induces eddy currents $\mathbf{J}$ in the surface layers of the testing material, and the interaction between the static magnetic field $\mathbf{B}$ and eddy currents $\mathbf{J}$ produces a Lorentz stress $\mathbf{F}$ based on Equation (1), which in turn generates ultrasound waves propagating within the testing sample.

$$
\mathbf{F}=\mathbf{J} \times \mathbf{B}
$$

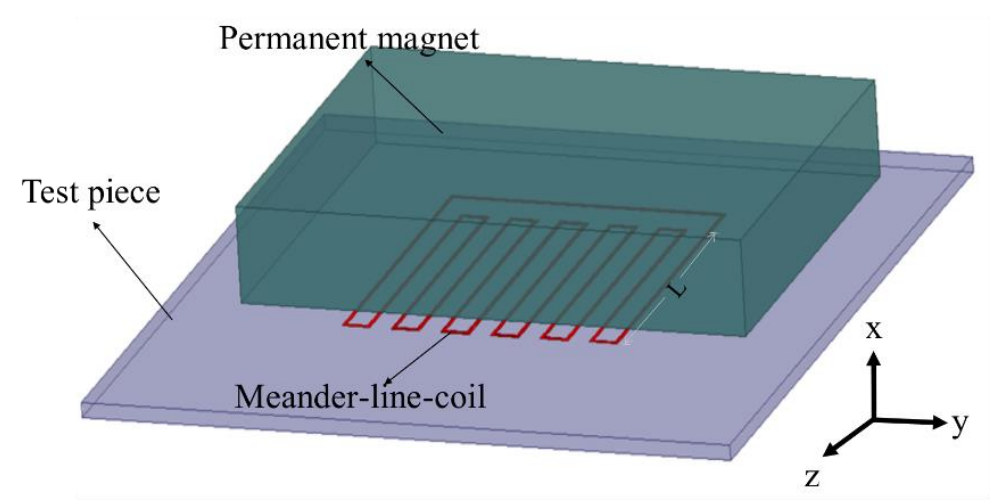

Figure 1. The configuration of a typical meander-line-coil EMAT.

Considerable works were reported on the electromagnetic acoustic transducers (EMATs) simulation, which contained the electromagnetic model and the ultrasonic model [20-23]. In addition, significant works were reported on the effect of parameters, such as the dimension of the coil, the dimension of the magnet, and the lift-off, on the EMATs efficiency for the optimal design of EMATs [24-27]. Because 3-D modelling has a high demand of the computer capacity and requires significant running time, most of the previous work were 2-D simulation focusing on the $x-y$ plane (i.e. vertical plane) of the material. The orientation of the coordinate system is shown in Figure 1, and all of the subsequent simulations are based on this coordinate system. Two examples of the 2-D modelling methods for the $x-y$ plane of the material have been detailed in [28, 29], however, there is little research on the Rayleigh waves' beam directivity on the surface of the material, that is, the $y-z$ plane of the material [30].

Consequently, this paper proposes a wholly analytical modelling method to study the Rayleigh waves' beam directivity on the surface of the material. This method involves the coupling of two models: an analytical electromagnetic (EM) model and an analytical ultrasonic (UT) model. The analytical EM model is used to calculate Lorentz force density, which then feed through to the analytical ultrasonic model to study the Rayleigh waves' beam directivity. In addition, the application of this model to the quantitative evaluation of the effect of the length of the meander-line-coil (" $L$ " in Figure 1) was conducted, and experiments were carried out to validate the simulation results.

\section{EMAT modelling}

In this work, the test piece used is an aluminium plate with a dimension of $600 \mathrm{~mm} \times 600$ $\mathrm{mm} \times 25 \mathrm{~mm}$; the coil used is a meander-line-coil with a dimension of $30 \mathrm{~mm} \times 34.163 \mathrm{~mm}$ $\times 0.035 \mathrm{~mm}$; the permanent magnet used is NdFeB35, whose size is $60 \mathrm{~mm} \times 60 \mathrm{~mm} \times 25 \mathrm{~mm}$. The operation frequency is $483 \mathrm{kHz}$, and the skin depth calculated is $0.117 \mathrm{~mm}$. The velocity of Rayleigh waves used in the aluminium plate is $2.93 \mathrm{~mm} / \mu \mathrm{s}$, so the spacing between two adjacent lines of the meander-line-coil is $3.03 \mathrm{~mm}$, which is equalling to one half of the Rayleigh waves' wavelength, to form the constructive interference. The meander-line-coil 
carries an alternating current with the peak of $5 \mathrm{~A}$, and the lift-off of the meander-line-coil is 1 $\mathrm{mm}$.

\subsection{EM simulation}

\subsubsection{Adapted analytical solution}

The analytical EM solution is adapted from the classic Deeds and Dodd solution, which was originally for the circular coil. This adapted analytical solution proposed by authors has been published in [28]; a brief introduction is presented here. The governing equations for the eddy current phenomena is shown in Equation(2)-(4), where $\mathbf{A}$ is the magnetic vector potential, $\mu$, $\sigma$ and $\varepsilon$ are the permeability, conductivity and permittivity of the material respectively, $\mathbf{I}$ is the applied current density, $\omega$ is the angular frequency of the applied alternating current, $\mathbf{E}$ is the electric field, and $\mathbf{J}$ is the induced eddy current [31].

$$
\begin{gathered}
\nabla^{2} \mathbf{A}=-\mu \mathbf{I}+\mu \sigma \frac{\partial \mathbf{A}}{\partial t}+\mu \varepsilon \frac{\partial^{2} \mathbf{A}}{\partial t^{2}}+\mu \nabla\left(\frac{1}{\mu}\right) \times(\nabla \times \mathbf{A}) \\
\mathbf{E}=-j \omega \mathbf{A} \\
\mathbf{J}=\sigma \mathbf{E}
\end{gathered}
$$

For a circular coil with a rectangular cross-section over a layered conductor, [14] provided the final analytical solutions for the vector potential calculation, which can be extended to calculate other electromagnetic induction phenomenon. Assuming the conductor only has one layer (as shown in Figure 2), the solutions can be simplified as:

$$
\begin{gathered}
\mathbf{A}(y, x)=\mu_{0} \mathbf{I} \int_{0}^{\infty} \frac{1}{a^{2}}\left(\int_{a r_{1}}^{a r_{2}} \gamma J_{1}(\gamma) d \gamma\right) J_{1}(a y)\left(e^{-a l}-e^{-a(l+h)}\right) \frac{e^{\left(2 a_{1} x\right)}}{\left(a+a_{1}\right)} d a \\
a_{1}=\sqrt{a^{2}+j \omega \mu_{1} \sigma_{1}}
\end{gathered}
$$

where $\mathbf{A}(y, x)$ is the vector potential within the conductor, $\mu_{0}$ is the permeability of air, $\mathbf{I}$ is the applied current density, $\gamma$ and $a$ are the integration variables, $r_{1}$ and $r_{2}$ are the inside radius and outside radius of the coil respectively, $J_{1}(\gamma)$ and $J_{1}(a y)$ are the Bessel functions of first kind, $l$ is the lift-off, $h$ is the height of the coil, $\mu_{1}$ and $\sigma_{1}$ are the permeability and conductivity of the conductor respectively, and $\omega$ is the angular frequency.

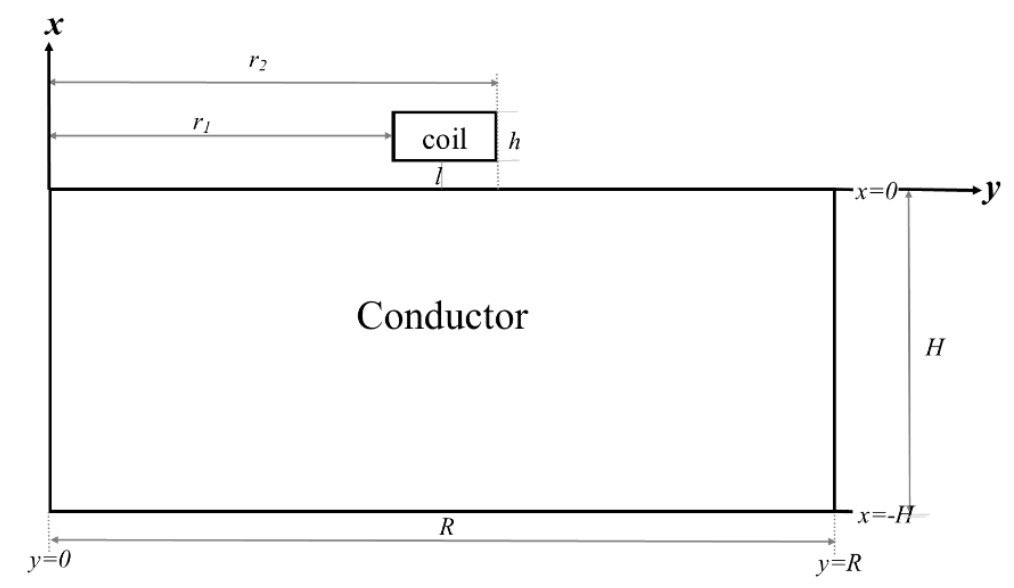

Figure 2. The geometry used for eddy current calculation.

An axis-symmetrical geometry, with detailed parameters shown in TABLE I, is built to study the analytical solution. Figure 3(a) illustrates the distribution of the vector potential along the 
surface of the aluminium plate $(x=0)$; the red square in this curve means the maximum vector potential. From this image, the vector potential is not symmetrical with $y=5 \mathrm{~mm}$, where the circular coil is located; that is because the wire of the circular coil is bent.

We proposed a hypothesis, that is, when the radius of the circular coil is large enough, the bent wire of the circular coil can be approximated to a straight wire, and the vector potential should be symmetrical. The model used is the same with the one used in TABLE I, with only one difference that the radius of the circular coil is enlarged to $10.04 \mathrm{~mm}$. Figure 3(b) shows the result of the vector potential distribution for a large-radius coil. The vector potential is now symmetrical with $\mathrm{y}=10.04 \mathrm{~mm}$; this verifies the hypothesis that, when the radius of the circular coil is very large, the bent wire of the circular coil can be approximated to a straight wire, and the magnitude distribution of the vector potential is symmetrical with the radius [28].

TABLE I. DETAILED PARAMTERS USED FOR THE ANALYTICAL MODELLING.

\begin{tabular}{|c|c|c|c|c|}
\hline \multirow{3}{*}{$\begin{array}{l}\text { Test piece } \\
\text { ( Aluminium ) }\end{array}$} & \multicolumn{4}{|c|}{ Parameters } \\
\hline & Length $(R)$ & Height $(H)$ & bility $\left(\mu_{1}\right)$ & Conductivity $\left(\sigma_{1}\right)$ \\
\hline & $10 \mathrm{~mm}$ & $3 \mathrm{~mm}$ & $10^{-6} \mathrm{H} / \mathrm{m}$ & 38000000 Siemens/m \\
\hline \multirow{5}{*}{$\begin{array}{l}\text { Coil used } \\
\text { (Circular coil) }\end{array}$} & \multicolumn{4}{|c|}{ Parameters } \\
\hline & Inside Radius $\left(r_{1}\right)$ & Outside Radius $\left(r_{2}\right)$ & $\begin{array}{c}\text { Mean Radius } \\
\left(r_{1}+r_{2}\right) / 2\end{array}$ & Applied Current Density(I) \\
\hline & $4.95 \mathrm{~mm}$ & $5.05 \mathrm{~mm}$ & $5 \mathrm{~mm}$ & $1 \mathrm{~A} / \mathrm{m}^{2}$ \\
\hline & Frequency $(f)$ & Lift-off $(l)$ & Height $(h)$ & Air permeability $\left(\mu_{0}\right)$ \\
\hline & $1 \mathrm{kHz}$ & $1 \mathrm{~mm}$ & $1 \mathrm{~mm}$ & $1.257 \times 10^{-6} \mathrm{H} / \mathrm{m}$ \\
\hline
\end{tabular}
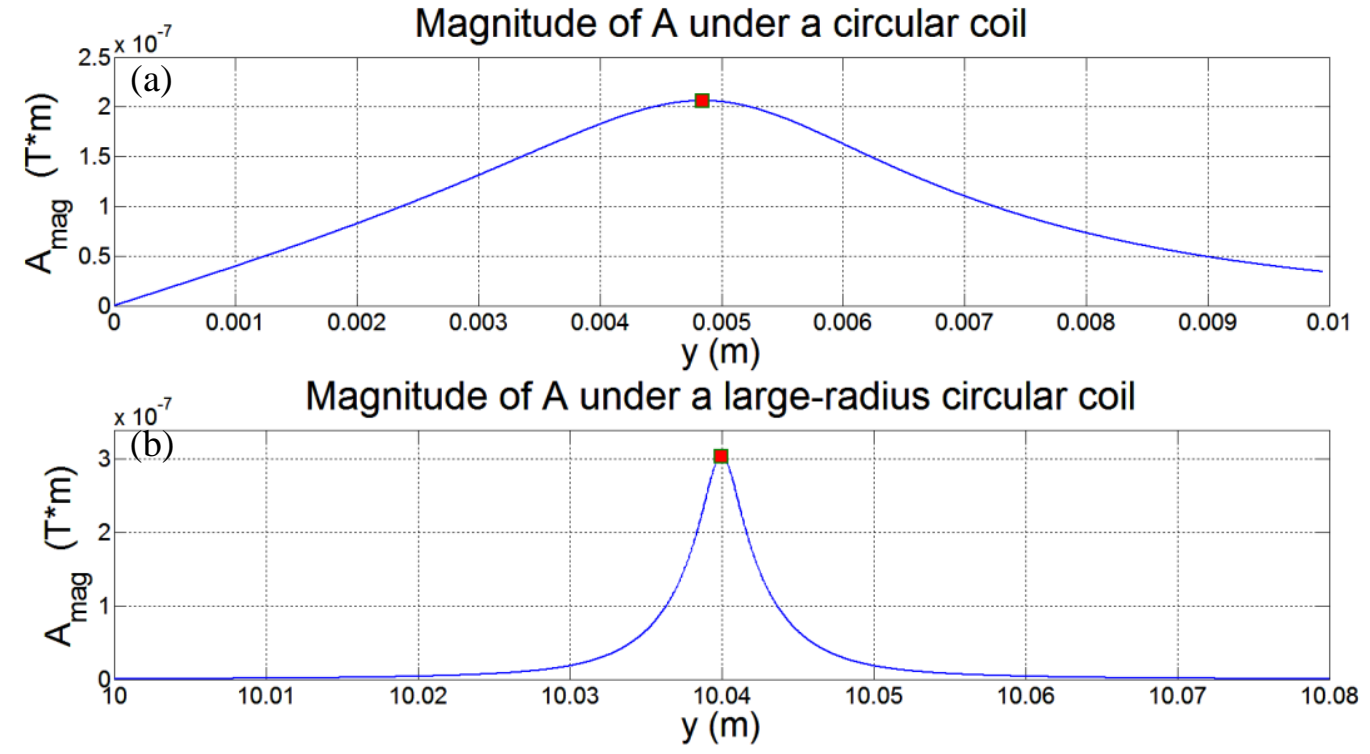

Figure 3. The vector potential distribution for a circular coil.

In order to analyse the accuracy of the proposed adapted analytical solutions, the finite element method (FEM) is employed to compare the results between the analytical and the numerical methods. Maxwell Ansoft based on the finite element method (FEM) is used to solve the vector potential; the model built with Maxwell Ansoft is the same with the one built in Figure 3(b). The computation of the FEM solver is based on minimising the energy error; 
the vacuum region to be solved is four times as larger as the aluminium plate; the mesh number used is over 7000, which makes the energy error below to $0.1 \%$. The comparison between the analytical method and the finite element method (FEM) is undertaken, not only at a low frequency but also at a high frequency. The comparison results are shown in Figure 4, revealing that the analytical method shows an accuracy benefit over the finite element method (FEM): the vector potential from the finite element method (FEM) does not approach to zero when it is far away from the wire (Figure 4(a),(b) and (c)); the curve from the finite element method (FEM) is not smooth at the high frequency, that is due to the numerical nature of the FEM: numerical approximation due to finite mesh density and element interpolation are inevitable.
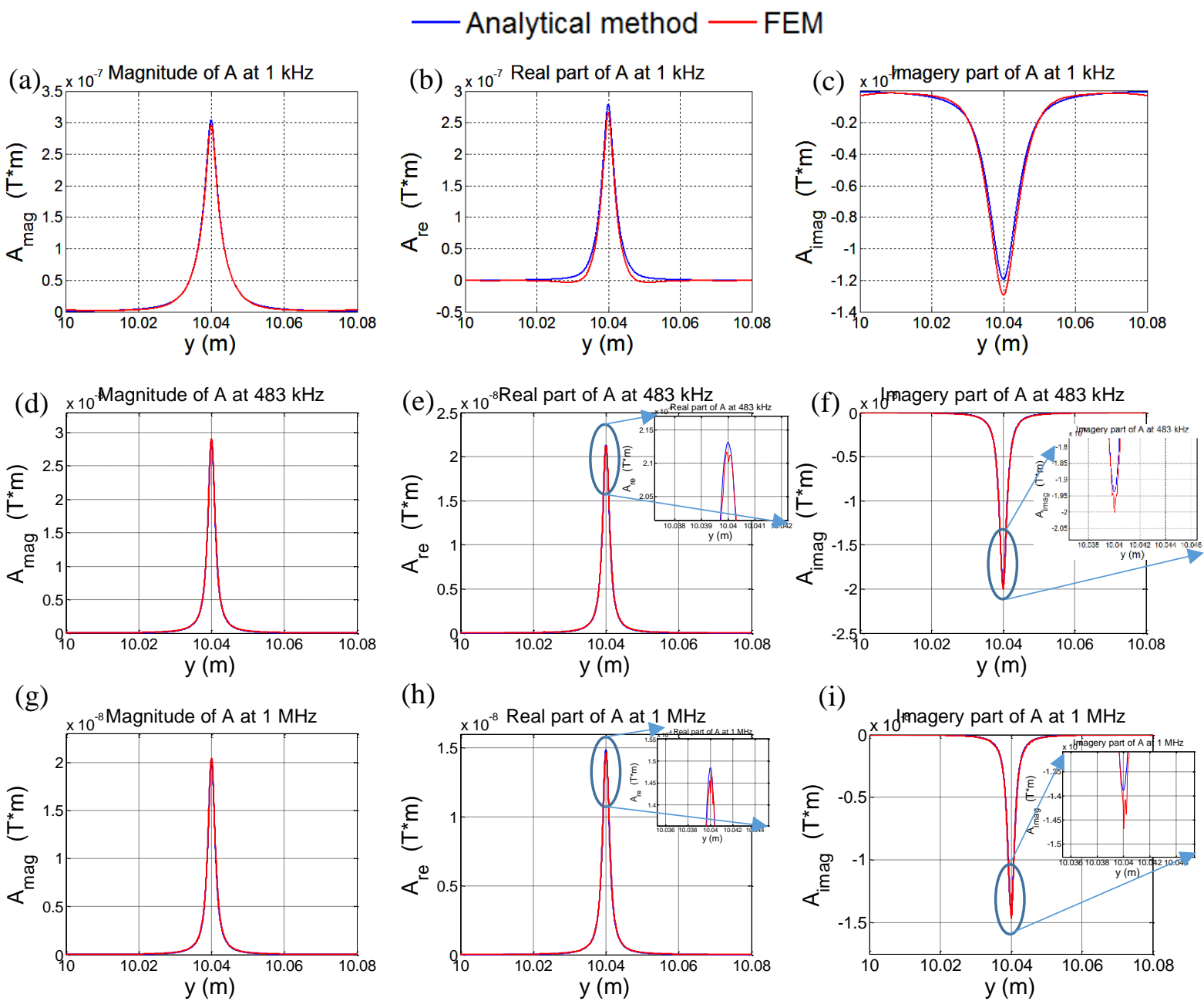

Figure 4. The comparison between the analytical method and the FEM method.

\subsubsection{EMAT-EM modelling}

As described previously, the analytical solution for a straight wire located above the aluminium plate has been studied. For a meander-line-coil, the total vector potential is the sum of the vector potential generated by each wire segment in the meander coil. Based on Equations (1)-(4), the Lorentz force density can be calculated.

The dimension of the used meander-line-coil is $30 \mathrm{~mm} \times 34.163 \mathrm{~mm} \times 0.035 \mathrm{~mm}$, which is very small compared to size of the test piece, $600 \mathrm{~mm} \times 600 \mathrm{~mm} \times 25 \mathrm{~mm}$. In order to improve the modelling efficiency, only the area, $100 \mathrm{~mm} \times 100 \mathrm{~mm} \times 10 \mathrm{~mm}$, where the 
meander-line-coil has a major effect on is selected to carry out the electromagnetic simulation.

The distribution of the vector potential and that of the Lorentz force density along the surface of the aluminium plate $(x=0)$ are shown in Figure 5(a) and Figure 5(b) respectively; the fields distribution between two adjacent wires are opposite due to the opposite alternating currents. Because there are six pairs of adjacent wires with opposite currents directions, both the curve of the vector potential and the curve of the Lorentz force density have 6 crests and 6 troughs. The value of the vector potential under the outmost wires is larger than that under the inner wires because the outmost vector potential are only affected by the fields on one side.
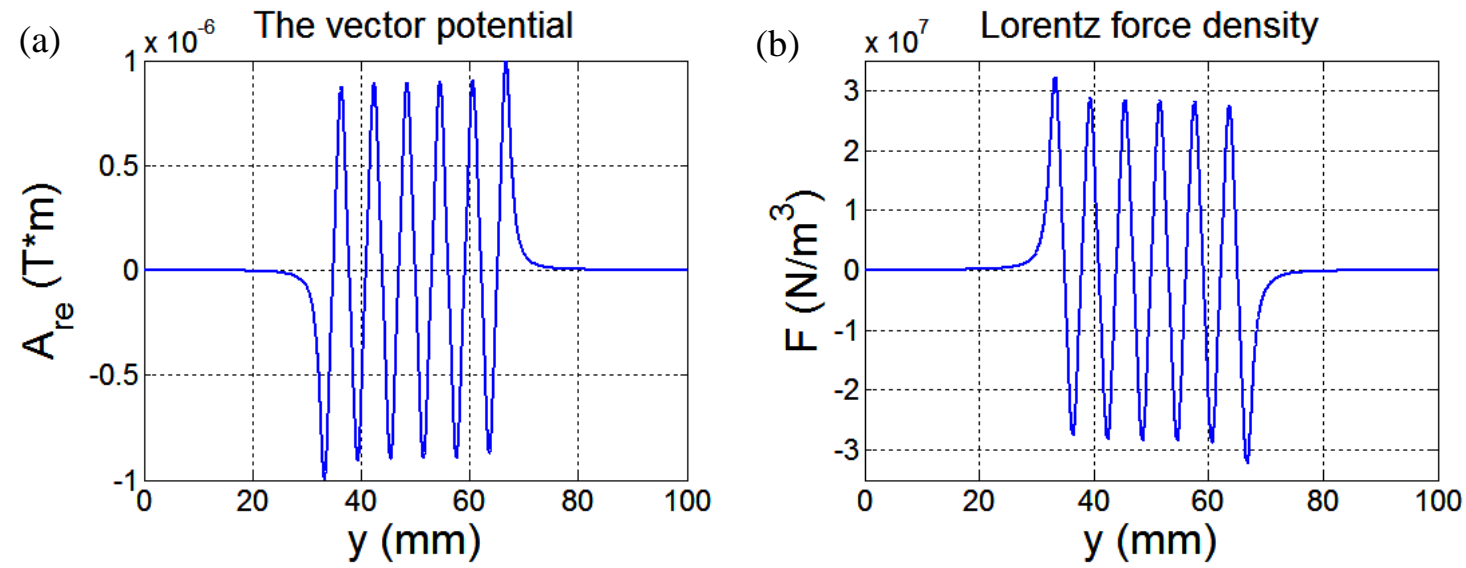

Figure 5. (a), the vector potential distribution along the surface of the material $(x=0)$; (b), the Lorentz force density distribution along the surface of the material $(x=0)$.

It should be noted that, for the EMATs modelling, the dynamic magnetic field was neglected due to the small excitation AC current (5 A) used in this work; The effect of the dynamic magnetic field generated by the meander-line-coil must be considered when the excitation current is large as it is one of the mechanisms to generate ultrasound waves; [32] suggested, when the excitation exceeds 528.9 A, the dynamic magnetic field plays a more significant role than the static magnetic field, which is generated by the permanent magnet. Therefore, the vertical forces, which are generated by the dynamic magnetic field and the eddy current, are neglected in this work.

\subsection{UT simulation}

\subsubsection{Rayleigh waves' displacement due to the point source}

The study of the Rayleigh waves' beam directivity on the surface of the material has not been widely reported. In this work, the Rayleigh waves' radiation pattern on the $y$ - $z$ plane of the aluminium plate is analysed. N.A.Haskell proposed the analytical solution to the Rayleigh waves' radiation pattern due to point sources in a homogenous medium and in a multi-layered medium, in 1963 and 1964 respectively [33, 34]. Elastic waves radiate in an unbounded medium expressed in Cartesian coordinates was given by Love [35]. However, Sezawa's cylindrical wave functions are the most convenient ways to impose the free surface boundary conditions; hence, the transformation between these two representations are necessary; the free surface conditions are imposed by vanishing the stress components at the free surface. Rayleigh waves' components are separated out by calculating the residue at Rayleigh pole $[33,34]$. 
The articles, [33, 34], were published in the field of seismology, and some terminology in the field of seismology, such as strike-slip faults and dip-slip faults are used in these articles[33, 34]. In this work, the driven source is the tangentially polarized Lorentz force, which is corresponding to the strike-slip fault; after some manipulations, the final solution to the Rayleigh waves' displacement due to the surface point source is,

$$
\begin{gathered}
\boldsymbol{u}_{\boldsymbol{r}}=A(\mathrm{\kappa}, r) e^{-\frac{\pi i}{4} \frac{2 \mathrm{k}(\gamma-1)}{v_{\beta}} \boldsymbol{F}\left(\frac{\gamma+1}{\gamma}-1\right)} \\
\boldsymbol{u}_{\boldsymbol{x}}=\frac{i \gamma v_{\alpha} \boldsymbol{u}_{r}}{\mathrm{k}(\gamma-1)}
\end{gathered}
$$

where

$$
\begin{gathered}
A(\mathrm{~K}, r)=\frac{\mathrm{K}^{2} \gamma v_{\beta}}{4 \rho\left(\frac{2 \gamma^{2} v_{\alpha} v_{\beta}}{\mathrm{K}^{3}}\right)} \sqrt{\frac{2}{\pi \mathrm{k} r}} e^{-i \mathrm{~K} r} \\
\gamma=\cos (\theta) \\
v_{\alpha}= \begin{cases}\sqrt{\mathrm{K}^{2}-\left(\omega / c_{L}\right)^{2}} \\
i \sqrt{\left(\omega / c_{L}\right)^{2}-\mathrm{K}^{2}} & \mathrm{~K}<\omega / c_{L}\end{cases} \\
v_{\beta}= \begin{cases}\sqrt{\mathrm{K}^{2}-\left(\omega / c_{S}\right)^{2}} & \mathrm{~K}>\omega / c_{L} \\
i \sqrt{\left(\omega / c_{S}\right)^{2}-\mathrm{K}^{2}} & \mathrm{~K}<\omega / c_{S}\end{cases} \\
\mathrm{K}=\frac{\omega}{c_{R}}
\end{gathered}
$$

where $\boldsymbol{u}_{\boldsymbol{r}}$ and $\boldsymbol{u}_{\boldsymbol{x}}$ are the in-plane displacement and out-of-plane displacement respectively. As shown in Figure 6, the arbitrary point on the surface of the material is defined as the field point; $r$ is the distance between the source point and the field point; $\mathrm{K}$ is the wavenumber of Rayleigh waves; $\boldsymbol{F}$ is the driven force; $\rho$ is the density of the material; $\theta$ is the angle between the force vector and the in-plane displacement vector; $\omega$ is the angular frequency; $c_{L}, c_{S}$ and $c_{R}$ are the velocity of the longitudinal wave, the shear wave and the Rayleigh wave respectively.

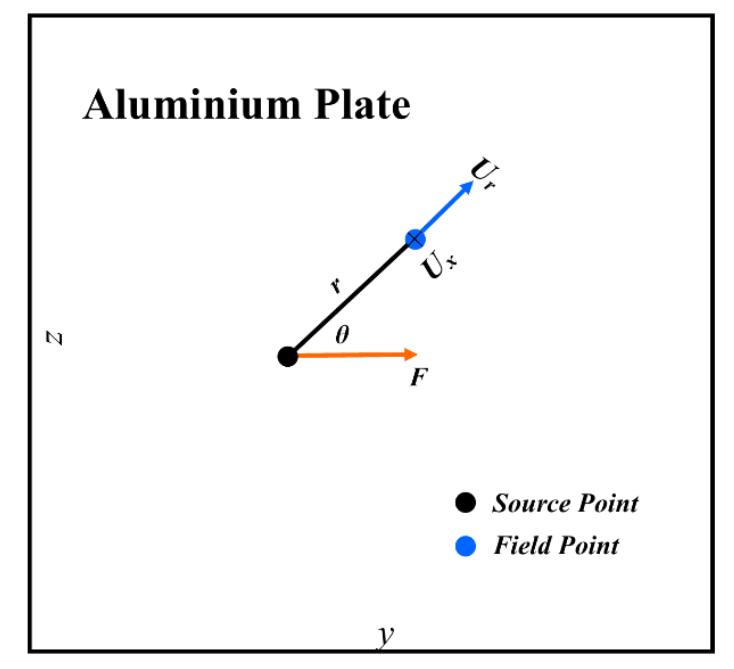

Figure 6. Surface waves generated by the point source. 


\subsubsection{EMAT-UT modelling}

Lorentz force densities, which are generated from the EM simulation, are the link between the EM simulation and the UT simulation, as shown in Figure 7. There are 12 straight wires in the meander-line-coil used in this work; therefore, there are 12 negative and positive peaks in the curve of the Lorentz force density distribution. These 12 Lorentz force densities are added to the UT simulation as the driven forces to generate Rayleigh waves. The displacement of Rayleigh waves due to a point source can be calculated (Equation (7)-(13)); with multiple point sources, the displacement of Rayleigh waves at an arbitrary field point is the sum of the displacement caused by each point source.

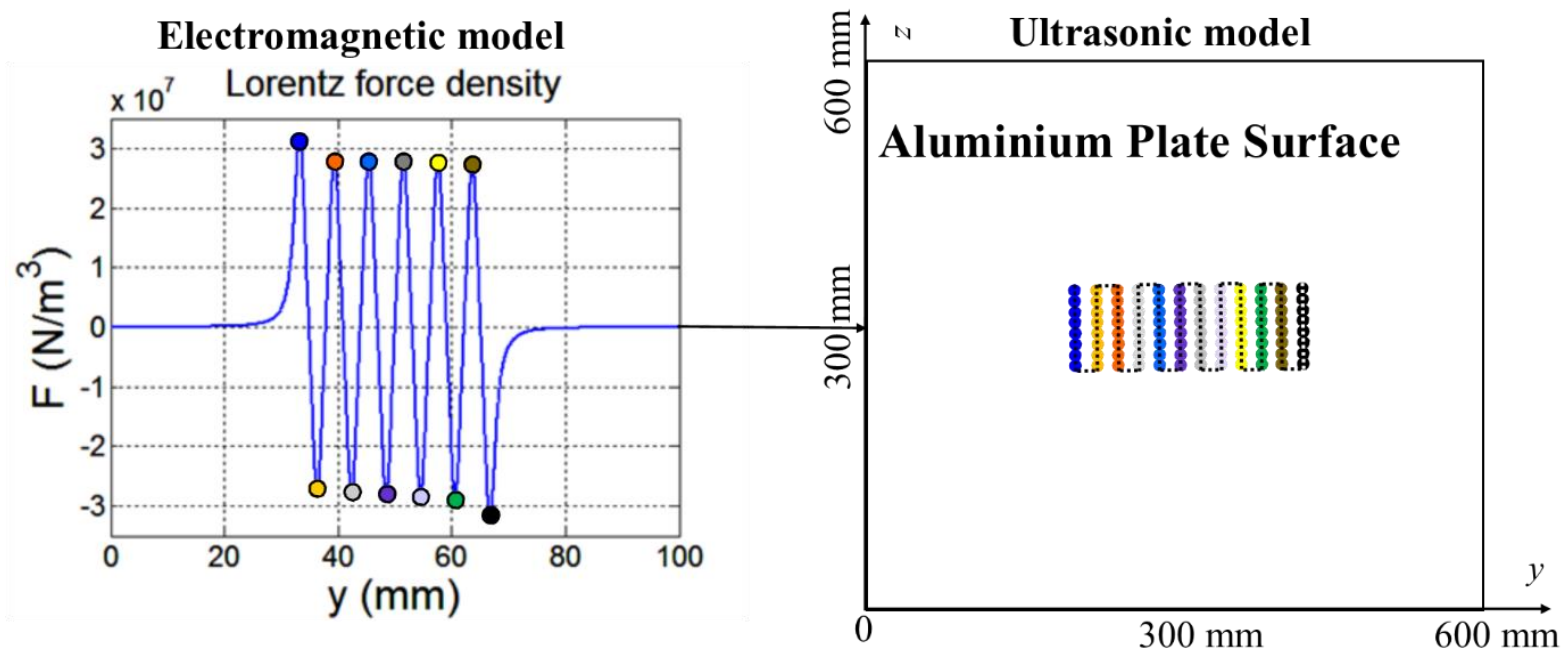

Figure 7. The transformation between the electromagnetic model and the ultrasonic model.

TABLE II illustrates the detailed parameters used for the EMAT-UT modelling. Field spatial step means the distance between two adjacent field points on the surface of the Aluminium plate; the dimension of the surface of the aluminium plate is $600 \mathrm{~mm} \times 600 \mathrm{~mm}$ and the field spatial step used is $1 \mathrm{~mm}$, so there are totally $601 \times 601$ field points on the surface of the Aluminium plate. Source spatial step for each wire means the distance between two adjacent source points on each wire; the length and the width of the wire is $30 \mathrm{~mm}$ and $0.8 \mathrm{~mm}$ respectively, and the source spatial step used is $0.2 \mathrm{~mm}$, so there are 151 source points along the wire length direction and 5 source points along the wire width direction on each wire. The reason we choose a dense source points is that lots of source points guarantee the wave interference' integrity.

TABLE II. DETAILED PARAMETERS USED FOR THE EMAT-UT MODELLING.

\begin{tabular}{lcc}
\hline Description & Symbol & Value \\
\hline Length of the Aluminum plate & $Y$ & $600 \mathrm{~mm}$ \\
Width of the Aluminum plate & $X$ & $600 \mathrm{~mm}$ \\
Field spatial step & $\Delta x_{f}$ & $1 \mathrm{~mm}$ \\
Length of the meander-line-coil & $L$ & $30 \mathrm{~mm}$ \\
Width of the meander-line-coil & $W$ & $0.8 \mathrm{~mm}$ \\
Source spatial step for each wire & $\Delta x_{s}$ & $0.2 \mathrm{~mm}$ \\
Density of the Aluminum plate & $\rho$ & $2700 \mathrm{~kg} / \mathrm{m}^{3}$ \\
Frequency & $f$ & $483 \mathrm{kHz}$ \\
Longitudinal waves' velocity & $C_{L}$ & $6.375 \mathrm{~mm} / \mu \mathrm{s}[32]$
\end{tabular}




\begin{tabular}{lll} 
Shear waves' velocity & $C_{s}$ & $3.14 \mathrm{~mm} / \mu \mathrm{s}[32]$ \\
Rayleigh waves' velocity & $C_{R}$ & $2.93 \mathrm{~mm} / \mu \mathrm{s}[32]$ \\
\hline
\end{tabular}

The meander-line-coil is located on the centre of the aluminium plate, so the whole EMATUT model is symmetrical with $y=300 \mathrm{~mm}$; in order to improve the modelling time, only the left half of the geometry is modelled. Only the area, $y$ from 0 to $250 \mathrm{~mm}$, is presented where the signals are significant. The calculated Rayleigh waves' radiation pattern is shown in Figure 8; the radiation pattern is consisting of the maximum displacement, including the inplane displacement and the out-of-plane displacement, at each field point. From this image, the Rayleigh waves are mainly concentrated along the $y$ direction. The area with larger intensities is referred to as the main lobe while the area with smaller intensities is referred to as the side lobe, as shown in Figure 8.

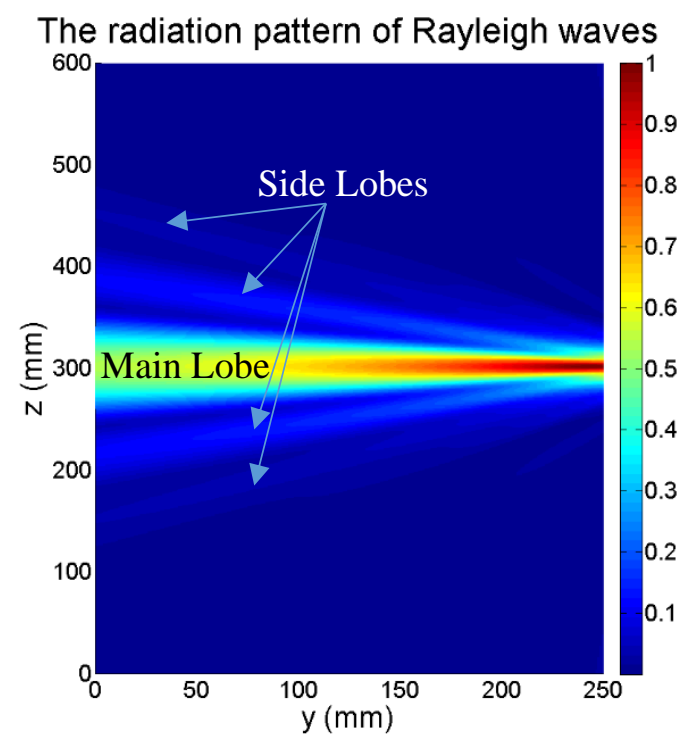

Figure 8. The Rayleigh waves' radiation pattern on the surface of the aluminium plate.

The whole radiation pattern is shown in Figure 9; as mentioned previously, it is symmetrical with $y=300 \mathrm{~mm}$. Beam directivity, which are used to quantitatively analyse the Rayleigh waves' distribution, can be obtained on the basis of the radiation pattern of Rayleigh waves. As shown in the red arc in Figure 9, beam directivity is, at a specific distance (" $r$ " in Figure 9) from the centre of the EMAT sensor, the displacement distribution of Rayleigh waves. In this work, $r$ used is $250 \mathrm{~mm} ; \theta_{1}$ and $\theta_{2}$ used are $-70^{\circ}$ and $70^{\circ}$ respectively. 


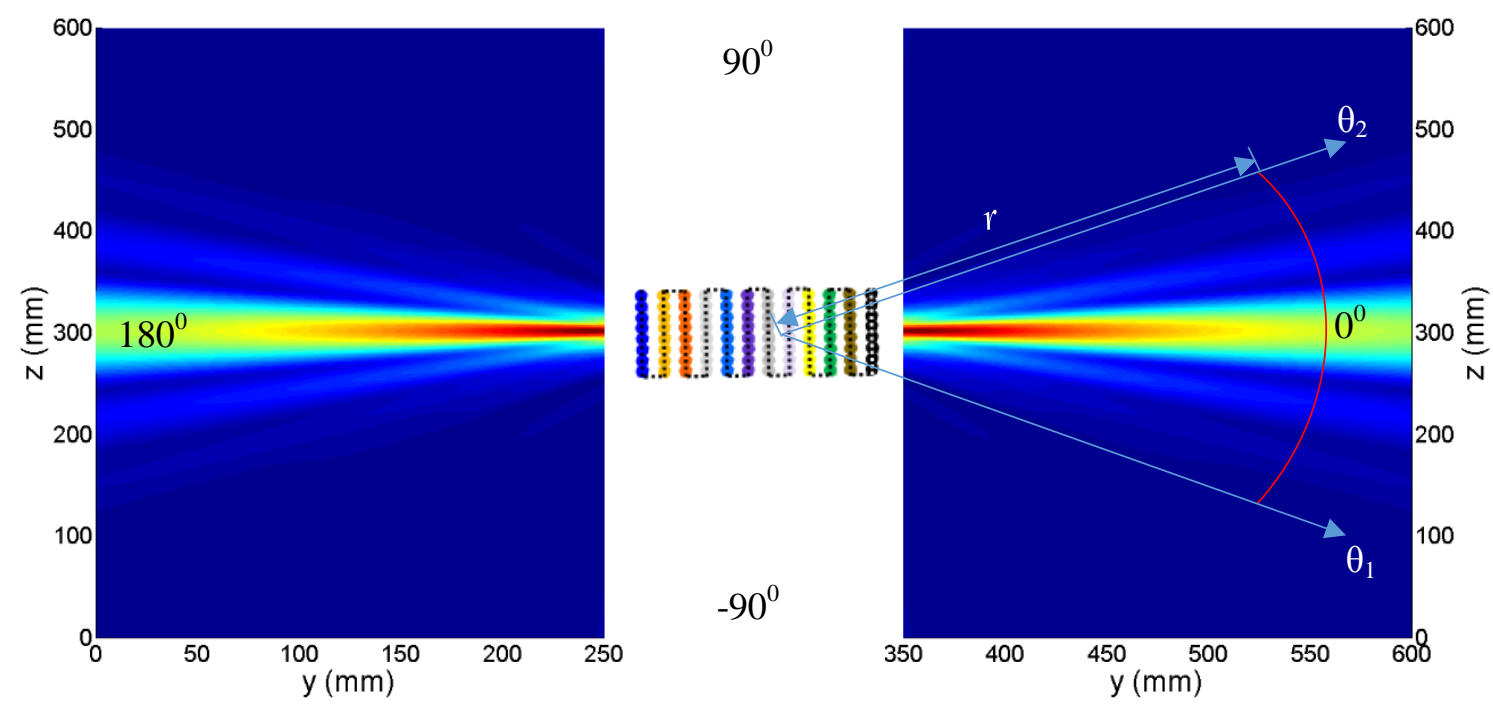

Figure 9. The model used to study the beam directivity.

The beam directivity is shown in Figure 10(a); this curve states the normalised magnitude of the displacement versus angles. As observed, there is a main lobe containing a larger displacement magnitude and some side lobes with a smaller displacement magnitude. The main lobe is centred at $0^{0}$, and the side lobes are roughly centred at $-30.5^{0},-18.5^{0}, 18.5^{0}$ and $30.5^{0}$ respectively. The side lobes are usually the radiation in undesired directions.

In order to quantitatively analyse the beam directivity, the half power beamwidth (HPBW) is employed. The half power beamwidth (HPBW) is the angle between the half-power $(-3 \mathrm{~dB})$ points of the main lobe; the half-power $(-3 \mathrm{~dB})$ points are the points with a magnitude equalling to a half of the peak value of the main lobe, as shown in Figure 10(b). In this work, for the meander-line-coil with a length of $30 \mathrm{~mm}$, the half power beamwidth (HPBW) is $14.94^{0}$.
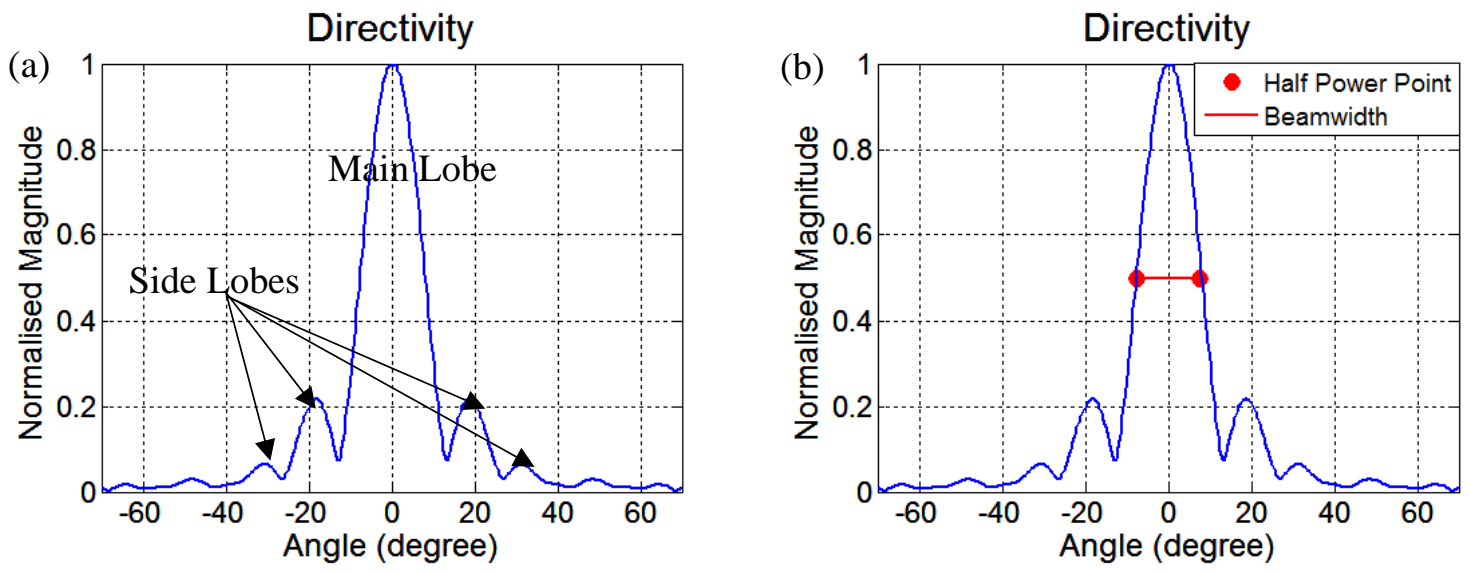

Figure 10. The directivity of Rayleigh waves for a 30mm-length meander-line-coil.

\subsection{The effect of the length of the meander-line-coil on radiation pattern}

In this section, the length effect of the meander-line-coil on the Rayleigh waves' radiation pattern is studied. Various lengths of the meander-line-coil were modelled; the modelling parameters are the same with those used in TABLE II except the length of the coil. The 
length of the meander-line-coil is from $20 \mathrm{~mm}$ to $45 \mathrm{~mm}$ with a step of $5 \mathrm{~mm}$; the beam directivity is shown in Figure 11.

Figure 11(a) states the beam directivity of the meander-line-coil with a length of $10 \mathrm{~mm}, 15$ $\mathrm{mm}, 20 \mathrm{~mm}$ and $25 \mathrm{~mm}$ respectively; from this image, with a larger length, the main lobe is narrower. It means, for the meander-line-coil with a larger length, it has a more concentrated Rayleigh waves' beam. The observation is further verified by Figure 11(b), which shows the beam directivity for the meander-line-coil with a length of $30 \mathrm{~mm}, 35 \mathrm{~mm}, 40 \mathrm{~mm}$ and 45 mm respectively.
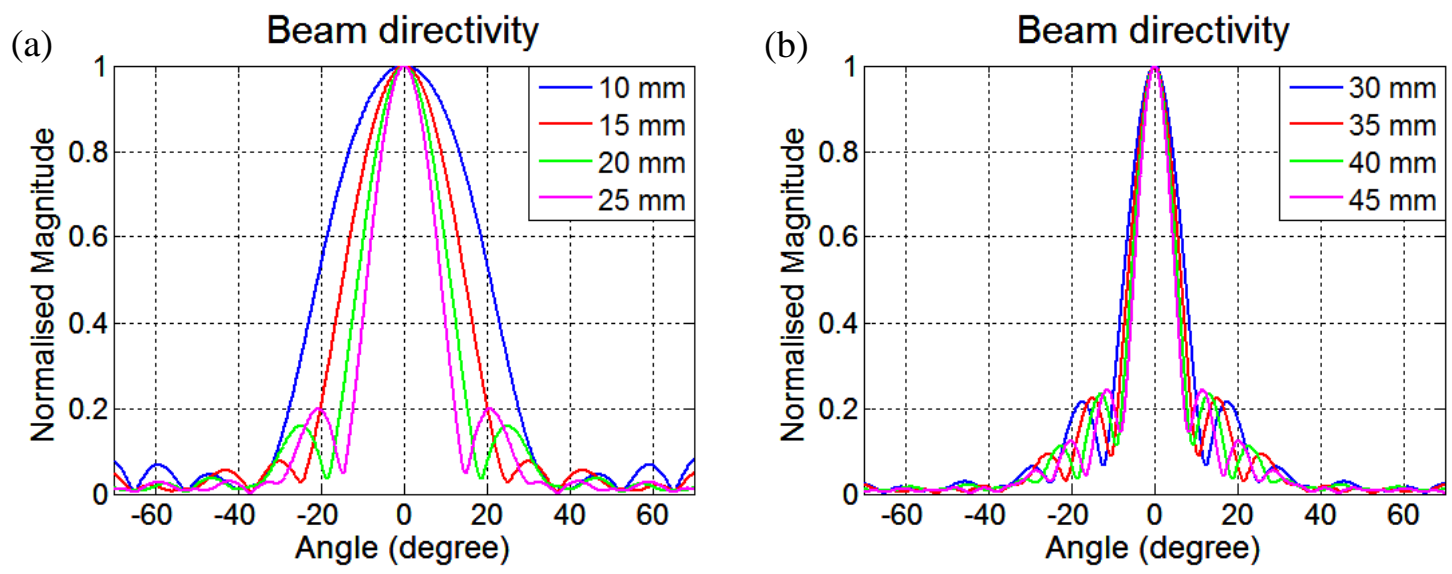

Figure 11 . The beam directivity for the meander-line-coil with various lengths.

The half power beamwidth (HPBW) for the meander-line-coil with various lengths is shown in TABLE III; it illustrates that a larger length leading to a smaller beamwidth. When the length of the meander-line-coil is enlarged from $10 \mathrm{~mm}$ to $45 \mathrm{~mm}$, the beamwidth decreases by 23.99 percent.

TABLE III. BEAMWIDTH AT VARIOUS LENGTHS.

\begin{tabular}{ccc|cc}
\hline & Length & Beamwidth & Length & Beamwidth \\
\cline { 2 - 5 } Meander-line-coil & $10 \mathrm{~mm}$ & $41.68^{0}$ & $30 \mathrm{~mm}$ & $14.94^{0}$ \\
& $15 \mathrm{~mm}$ & $29.63^{0}$ & $35 \mathrm{~mm}$ & $12.96^{0}$ \\
& $20 \mathrm{~mm}$ & $22.24^{0}$ & $40 \mathrm{~mm}$ & $11.32^{0}$ \\
& $25 \mathrm{~mm}$ & $17.92^{0}$ & $45 \mathrm{~mm}$ & $10^{0}$ \\
\hline
\end{tabular}

\section{Experimental results}

Experiments were carried out to validate simulations; the experimental set-up is shown in Figure 12(a); the aluminium plate, the meander-line-coil and the permanent magnet used have the same dimension and shape with the modelling geometry as clarified in the EMAT modelling section. The high power tone burst pulser and receiver, RITEC RPR4000, was used to excite and receive EMAT signals; an impedance matching box was used to match the impedance between the power amplifier and the coil to maximize the power transfer; oscilloscope is to display and record signals. Data can be sent to the external computer to process either by RITEM RPR4000 or by the oscilloscope. 

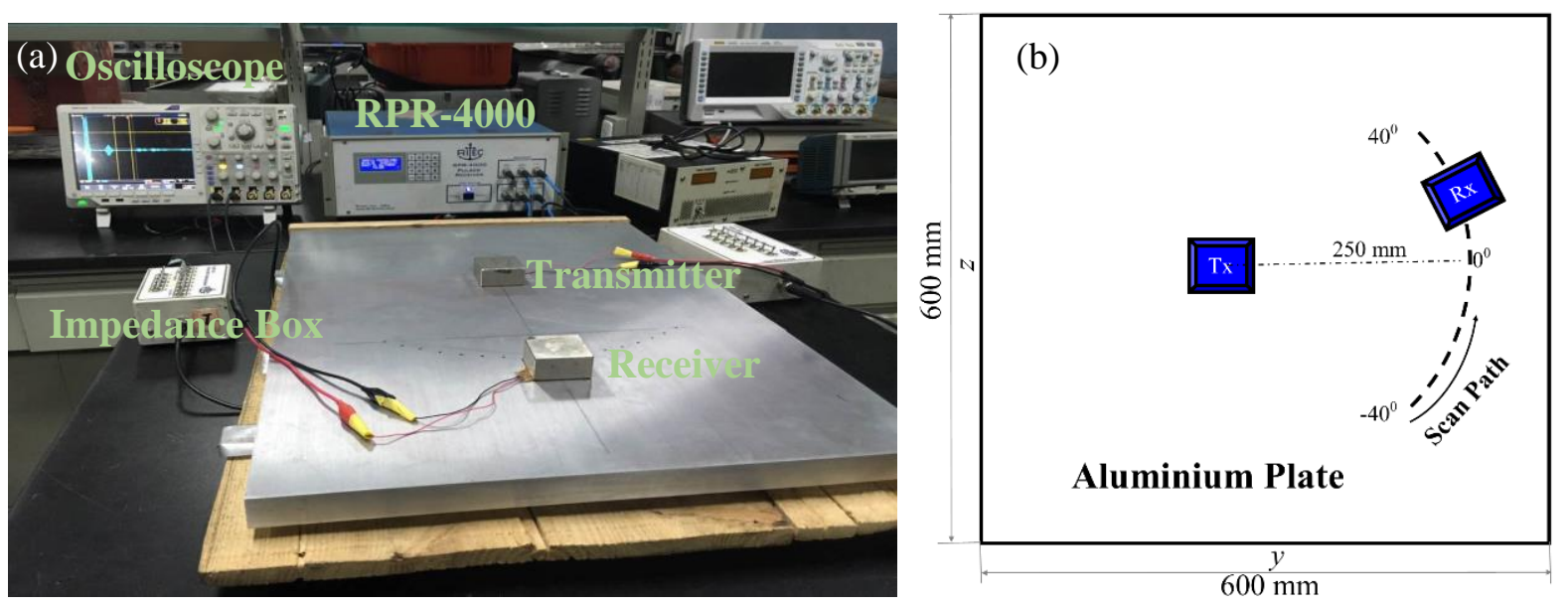

Figure 12. (a), the experiments set-up; (b), the scan path of the receiver.

In EMAT modelling, the length range of the meander-line-coil used is from $10 \mathrm{~mm}$ to $45 \mathrm{~mm}$ with a step of $5 \mathrm{~mm}$. For experiments, in order to reduce the experimental complexity, only four lengths, $10 \mathrm{~mm}, 20 \mathrm{~mm}, 30 \mathrm{~mm}$ and $40 \mathrm{~mm}$, are fabricated to validate the EMAT modelling. The scan path of the receiver is shown in Figure 12(b); the centre-to-centre distance between the transmitter and the receiver is $250 \mathrm{~mm}$; the scan path is from $-40^{\circ}$ to $40^{\circ}$ with a step of $2.5^{0}$. The meander-line-coil receiver used is with a length of $10 \mathrm{~mm}$; by moving the receiver along the scanning path, the directly transmit Rayleigh waves can be received by the receiver at different angles.

The measured beam directivity from the experiment is shown in Figure 13; the signals are normalised by the peak value among all of the measured data. Because the receiver is moved from $-40^{\circ}$ to $40^{\circ}$ with a step of $2.5^{\circ}$, only 33 sampling points are recorded, leading to a coarse curve of the measured beam directivity. The experimental results suggest that with a larger length, the Rayleigh waves' beam has a more concentrated main lobe; while with a smaller length, the Rayleigh waves are distributed; these results are consistent with the modelling results.

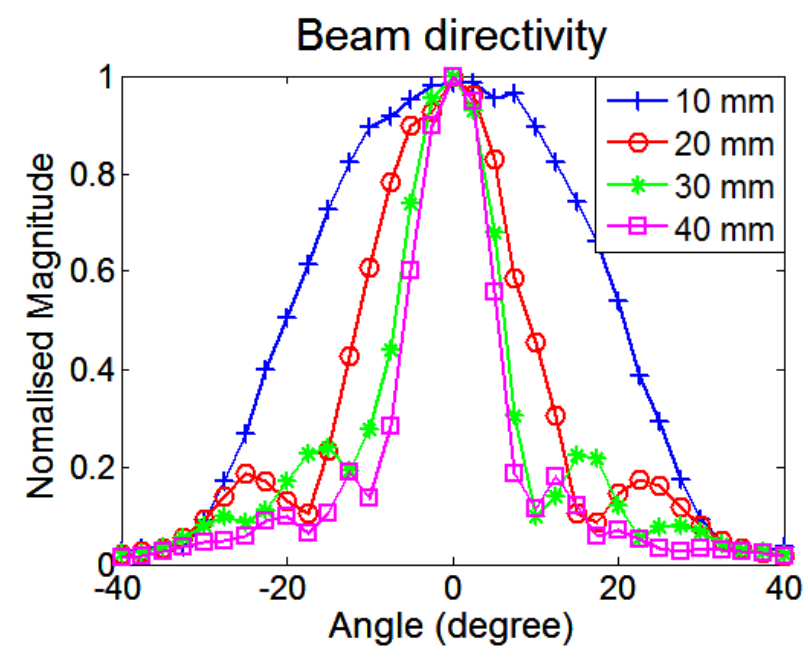

Figure 13. The measured beam directivity from experiments.

Figure 14 shows the comparison between the measured results and the simulated results. From Figure 14(a), for the meander-line-coil with a length of $10 \mathrm{~mm}$, the measured beam directivity is consistent with the simulated beam directivity. For the meander-line-coil with a length of $20 \mathrm{~mm}, 30 \mathrm{~mm}$ and $40 \mathrm{~mm}$, there are some non-consistent points between the 
measured and simulated curve; however, the trend of the measured curve is the same with that of the simulated curve. Possible reasons for the non-consistent points are: 1), the inevitable noises of experiments; 2), the errors due to the positioning of the receiver. Overall, the measured beam directivity shows a good agreement with the simulated beam directivity.
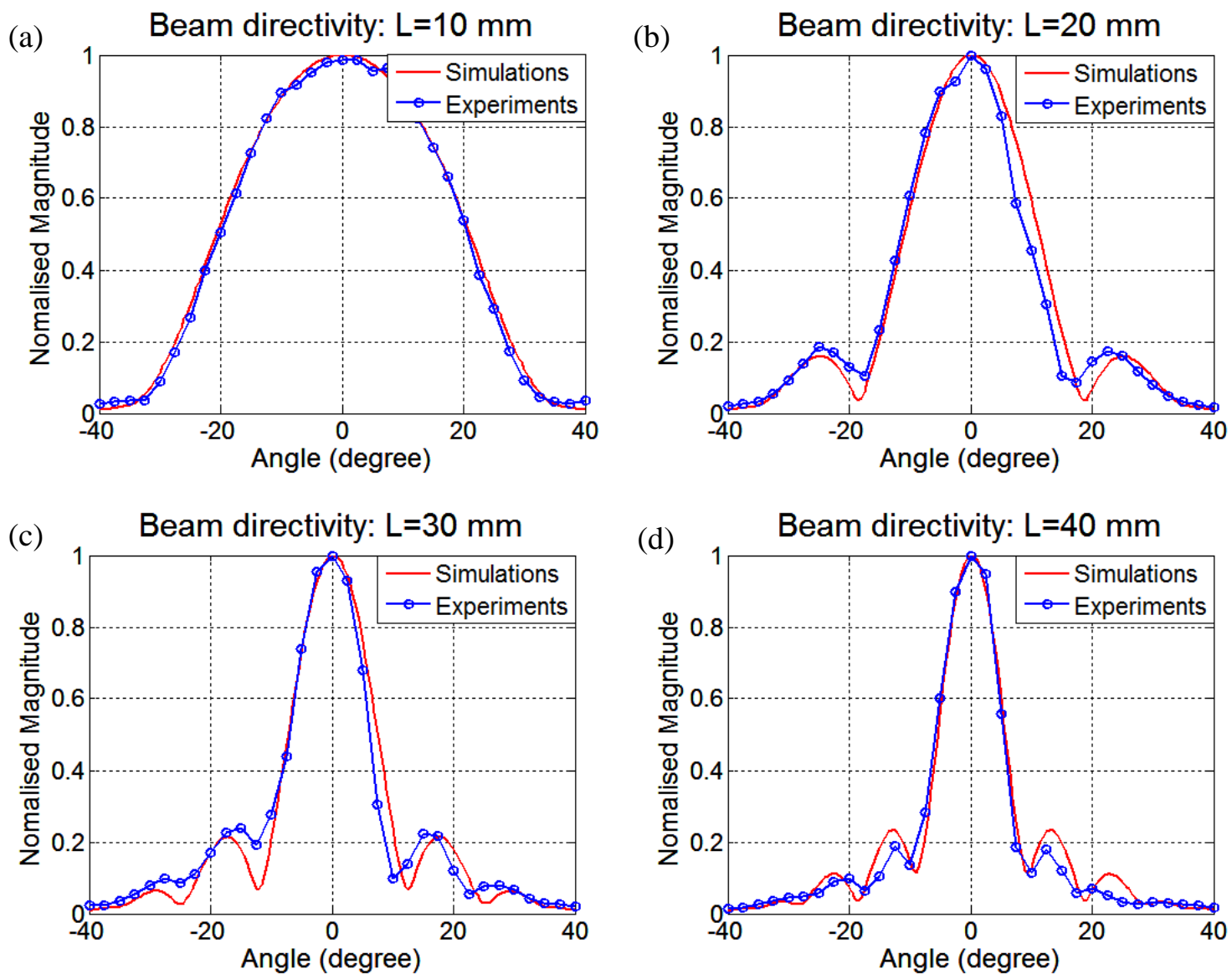

Figure 14. Comparison between the simulated and measured results for the meander-line-coil with a length of $10 \mathrm{~mm}, 20 \mathrm{~mm}, 30 \mathrm{~mm}$ and $40 \mathrm{~mm}$ respectively.

\section{Conclusion}

A wholly analytical method to build EMAT models to study the Rayleigh waves' beam directivity is proposed. For the EM simulation, the EM analytical solution to the meanderline-coil is adapted from the classic Deeds and Dodd solution, which was originally intended for circular coils. The adapted analytical method is verified by the finite element method (FEM). For the UT simulation, an analytical ultrasonic model is built to calculate the Rayleigh waves' displacement produced by the Lorentz force densities. A semi-analytical technique, named distributed point source method (DPSM), was introduced by Placko and Kundu in 2001 [36], and it has been proved that DPSM is a highly efficient method for solving EMAT problems with the advantage of less mesh size over FEM and guarantees good results for the non-flat transducer face $[37,38]$. It will be worth comparing the results based on FEM and those based on DPSM in the future.

Modelling results reveal that, for a meander-line-coil, the length of the meander-line-coil has a crucial effect on the Rayleigh waves' beam directivity; that is, a larger length results in a narrower main lobe, which means the Rayleigh waves are more concentrated. However, the larger-length meander-line-coil are on the sacrifice of the sensor geometry; in other words, 
the larger-length meander-line-coil produce a more concentrated Rayleigh waves' beam but they are not desirable in applications where only small sensors are accessible.

Four meander-line-coils, with a length of $10 \mathrm{~mm}, 20 \mathrm{~mm}, 30 \mathrm{~mm}$ and $40 \mathrm{~mm}$ respectively, are fabricated and used to validate the proposed modelling method; the experimental results show a good agreement with the simulated results. In order to increase the spatial resolution of the received signal, a miniature receiver should be considered; [39] employed a miniature receiver, Pinducer, which is a displacement sensor with elements of 0.053 inch diameters, to receive the Rayleigh waves generated by the meander-line-coil EMATs; it is worthwhile trying this miniature receiver in the future. Overall, this study provides quantitative analysis of the effect of the length of the meander-line-coil on the Rayleigh waves' beam directivity.

\section{Acknowledgement}

This work was financially supported by Engineering and Physical Sciences Research Council (Grant No. EP/M020835/1).

\section{References}

[1] S. Dixon, C. Edwards, and S. B. Palmer, "A laser-EMAT system for ultrasonic weld inspection," Ultrasonics, vol. 37, pp. 273-281, 1999.

[2] N. Korde and T. Kundu, "Material hardness and ageing measurement using guided ultrasonic waves," Ultrasonics, vol. 53, pp. 506-510, 2013.

[3] M. Lovejoy, Magnetic particle inspection: a practical guide: Springer Science \& Business Media, 2012.

[4] W. Yin and A. Peyton, "Thickness measurement of non-magnetic plates using multifrequency eddy current sensors," NDT \& E International, vol. 40, pp. 43-48, 2007.

[5] W. Yin and A. J. Peyton, "Thickness measurement of metallic plates with an electromagnetic sensor using phase signature analysis," Instrumentation and Measurement, IEEE Transactions on, vol. 57, pp. 1803-1807, 2008.

[6] P. Cawley, "Ultrasonic measurements for the quantitative NDE of adhesive joints-potential and challenges," in Ultrasonics Symposium, 1992. Proceedings., IEEE 1992, 1992, pp. 767-772.

[7] B. Chassignole, D. Villard, M. Dubuget, J. Baboux, and R. El Guerjouma, "Characterization of austenitic stainless steel welds for ultrasonic NDT," in REVIEW OF PROGRESS IN QUANTITATIVE NONDESTRUCTIVE EVALUATION: Volume 19, 2000, pp. 1325-1332.

[8] B. Drinkwater and P. Cawley, "Measurement of the frequency dependence of the ultrasonic reflection coefficient from thin interface layers and partially contacting interfaces," Ultrasonics, vol. 35, pp. 479-488, 1997.

[9] B. W. Drinkwater and P. D. Wilcox, "Ultrasonic arrays for non-destructive evaluation: A review," NDT \& E International, vol. 39, pp. 525-541, 2006.

[10] J. Gallego-Juarez, "Piezoelectric ceramics and ultrasonic transducers," Journal of Physics E: Scientific Instruments, vol. 22, p. 804, 1989.

[11] H. Jaffe and D. Berlincourt, "Piezoelectric transducer materials," Proceedings of the IEEE, vol. 53, pp. 1372-1386, 1965.

[12] A. Sinclair and A. Chertov, "Radiation endurance of piezoelectric ultrasonic transducers-A review," Ultrasonics, vol. 57, pp. 1-10, 2015.

[13] M. Akhnak, O. Martinez, L. Ullate, and F. M. de Espinosa, "64 elements two-dimensional piezoelectric array for 3D imaging," Ultrasonics, vol. 40, pp. 139-143, 2002.

[14] C. Scruby and B. Moss, "Non-contact ultrasonic measurements on steel at elevated temperatures," NDT \& E International, vol. 26, pp. 177-188, 1993. 
[15] R. Edwards, S. Dixon, and X. Jian, "Non - Contact Ultrasonic Characterization of Defects Using EMATS," in REVIEW OF PROGRESS IN QUANTITATIVE NONDESTRUCTIVE EVALUATION, 2005, pp. 1568-1575.

[16] R. Dhayalan and K. Balasubramaniam, "A hybrid finite element model for simulation of electromagnetic acoustic transducer (EMAT) based plate waves," NDT \& E International, vol. 43, pp. 519-526, 2010.

[17] P. J. Latimer and D. T. MacLauchlan, "EMAT probe and technique for weld inspection," ed: Google Patents, 1998.

[18] M. Hirao and $\mathrm{H}$. Ogi, EMATs for science and industry: noncontacting ultrasonic measurements: Springer Science \& Business Media, 2003.

[19] R. Dhayalan and K. Balasubramaniam, "A two-stage finite element model of a meander coil electromagnetic acoustic transducer transmitter," Nondestructive Testing and Evaluation, vol. 26, pp. 101-118, 2011.

[20] X. Jian, S. Dixon, K. Grattan, and R. S. Edwards, "A model for pulsed Rayleigh wave and optimal EMAT design," Sensors and Actuators A: Physical, vol. 128, pp. 296-304, 2006.

[21] L. Kang, S. Dixon, K. Wang, and J. Dai, "Enhancement of signal amplitude of surface wave EMATs based on 3-D simulation analysis and orthogonal test method," NDT \& E International, vol. 59, pp. 11-17, 2013.

[22] S. Wang, L. Kang, Z. Li, G. Zhai, and L. Zhang, "A novel method for modeling and analysis of meander-line-coil surface wave EMATs," in Life System Modeling and Intelligent Computing, ed: Springer, 2010, pp. 467-474.

[23] S. Wang, Z. Li, L. Kang, and G. Zhai, "Influence of Coil Parameters on Rayleigh Waves Excited by Meander-Line Coil EMATs," in Intelligent Computing for Sustainable Energy and Environment, ed: Springer, 2013, pp. 94-103.

[24] X. Chen, R. Su, H. Zhang, S. Wang, and G. Zhai, "Influence of coil parameters on transduction performance of unidirectional EMATs for Rayleigh wave," in Nondestructive Evaluation/Testing: New Technology \& Application (FENDT), 2013 Far East Forum on, 2013, pp. 150-154.

[25] L. Kang, F. Pan, R. Su, C. Zhang, and J. Dai, "A new surface-wave EMAT design of enhanced transduction efficiency," in Nondestructive Evaluation/Testing (FENDT), 2014 IEEE Far East Forum on, 2014, pp. 9-13.

[26] Y. Zhao, Z. Q. Jia, G. Rui, J. Ma, J. F. Song, J. H. Sun, and S. Liu, "A novel laser-EMAT system for noncontact testing metal materials," in Applied Mechanics and Materials, 2013, pp. 422425.

[27] S. Wang, L. Kang, P. Xin, and G. Zhai, "Characteristic research and analysis of EMAT's transduction efficiency for surface detection of aluminum plate," in Electronic Measurement \& Instruments, 2009. ICEMI'09. 9th International Conference on, 2009, pp. 4-949-4-955.

[28] Y. Xie, S. Rodriguez, W. Zhang, Z. Liu, and W. Yin, "Simulation of an Electromagnetic Acoustic Transducer Array by using Analytical method and FDTD," Journal of Sensors, vol. 501, p. 5451821, 2016.

[29] Y. Xie, W. Yin, Z. Liu, and A. Peyton, "Simulation of ultrasonic and EMAT arrays using FEM and FDTD," Ultrasonics, vol. 66, pp. 154-165, 2016.

[30] Y. Xie, L. Yin, R. G. Sergio, T. Yang, Z. Liu, and W. Yin, "A wholly analytical method for the simulation of an electromagnetic acoustic transducer array," International Journal of Applied Electromagnetics and Mechanics, pp. 1-15.

[31] C. Dodd and W. Deeds, "Analytical Solutions to Eddy - Current Probe - Coil Problems," Journal of applied physics, vol. 39, pp. 2829-2838, 1968.

[32] S. Wang, L. Kang, Z. Li, G. Zhai, and L. Zhang, "3-D modeling and analysis of meander-line-coil surface wave EMATs," Mechatronics, vol. 22, pp. 653-660, 2012.

[33] N. Haskell, "Radiation pattern of surface waves from point sources in a multi-layered medium," Bulletin of the Seismological Society of America, vol. 54, pp. 377-393, 1964. 
[34] N. Haskell, "Radiation pattern of Rayleigh waves from a fault of arbitrary dip and direction of motion in a homogeneous medium," Bulletin of the Seismological Society of America, vol. 53, pp. 619-642, 1963.

[35] A. Love, "H. A treatise on the mathematical theory of elasticity," Cambridge: Cambridge University Press, vol. 1, p. 952, 1944.

[36] D. Placko and T. Kundu, "Theoretical study of magnetic and ultrasonic sensors: dependence of magnetic potential and acoustic pressure on the sensor geometry," in 6th Annual International Symposium on NDE for Health Monitoring and Diagnostics, 2001, pp. 52-62.

[37] M. Eskandarzade, T. Kundu, N. Liebeaux, D. Placko, and F. Mobadersani, "Numerical simulation of electromagnetic acoustic transducers using distributed point source method," Ultrasonics, vol. 50, pp. 583-591, 2010.

[38] T. Kundu, Ultrasonic nondestructive evaluation: engineering and biological material characterization: CRC press, 2003.

[39] S. Wang, R. Su, X. Chen, L. Kang, and G. Zhai, "Numerical and experimental analysis of unidirectional meander-line coil electromagnetic acoustic transducers," IEEE transactions on ultrasonics, ferroelectrics, and frequency control, vol. 60, pp. 2657-2664, 2013. 